\title{
AXIOMS FOR NON-RELATIVISTIC QUANTUM MECHANICS
}

\author{
NEAL ZIERLER
}

Introduction. In the approach to the axiomatization of quantum mechanics of George W. Mackey [7], a series of plausible axioms is completed by a final axiom that is more or less ad hoc. This axiom states that a certain partially ordered set-the set $P$ of all two-valued observables-is isomorphic to the lattice of all closed subspaces of Hilbert space. The question arises as to whether this axiom can be deduced from others of a more a priori nature, or, more generally, whether the lattice of closed subspaces of Hilbert space can be characterized in a physically meaningful way. Our central result is a characterization of this lattice which may serve as a step in the indicated direction, although there is not now a precise sense in which our axioms are more plausible than his. Its principal features may be described as follows.

Suppose that $P$ is an atomic lattice, define an element to be finite if it is the join of a finite number of points, and suppose that the unit element is not finite, but is the join of a countable set of points. Suppose for the moment that

(F) The lattice under every finite element of $P$ is a real (or complex) projective geometry.

Then one additional axiom, which appears to be particularly mild from an operational viewpoint, is sufficient and necessary for us to show that $P$ is isomorphic to the lattice of closed subspaces of a separable, infinite dimensional real (or complex) Hilbert space.

Of course, (F) is not taken as an axiom, but is deduced from more primitive assumptions. This part of the development follows well-known lines, but the structure of $P$ (and its set $S$ of states) permits us to give it a rather simple form. For example, in order to conclude that the lattice under every finite element of $P$ is a projective geometry, we need make, in addition to the atomicity of $P$, only the following three assumptions: $P$ is not a Boolean algebra; the lattices under any pair of finite elements of the same dimension are isomorphic; a certain weak (and rather intuitive) form of the modular law holds under finite elements (Theorem 2.1).

In a preliminary chapter we examine the interrelation of a number of regularity properties which a pair $P, S$ satisfying a slight refinement of Mackey's basic axioms might have, and show that a few of the more

Received June 28, 1960. The work reported in this paper was performed while the author was a member of the staff of Lincoln Laboratory, Massachusetts Institute of Technology. He is now withe the Arcon Corporation, Lexington, Massachusetts. 
plausible properties imply all the others (Theorem 1.1).

This work is a modification of part of a thesis submitted to the Department of Mathematics of Harvard University in partial fulfillment of the requirements for the degree of Doctor of Philosophy.

1. Events and states: preliminaries. Let $P$ be a partly ordered set with least and greatest elements 0 and 1 respectively. If the greatest lower bound or least upper bound of elements $a$ and $b$ of $P$ exists in $P$ it is denoted $a b$ or $a \vee b$ respectively. Let $a \rightarrow a^{\prime}$ be an orthocomplementation in $P$; that is, for each $a \in P, a^{\prime} \in P$ and

(1) $\left(a^{\prime}\right)^{\prime}=a$,

(2) $a<b$ if and only if $b^{\prime}<a^{\prime}$,

(3) $a^{\prime}$ is a complement of $a$; i.e., $a^{\prime} a$ and $a \vee a^{\prime}$ exist and equal 0 and 1 respectively.

Two elements $a$ and $b$ of $P$ are said to be orthogonal, $a \perp b$, if and only if $a \leqq b^{\prime}$. Clearly $a \perp b$ is equivalent to $b \perp a$. If $Q$ is a set of pairwise orthogonal elements of $P$ we shall say, for short, that $Q$ is orthogonal. It is easy to see that De Morgan's law holds in $P:(a b)^{\prime}=$ $a^{\prime} \vee b^{\prime}$ in the sense that if either $a b$ or $a^{\prime} \vee b^{\prime}$ exists, so does the other and the equality holds.

We assume that $P$ satisfies

(L1) If $\left\{a_{1}, a_{2}, \cdots\right\}$ is orthogonal, then $\bigvee a_{i}$ exists in $P$.

It follows readily that a variety of sups and infs exists in $P$ : e.g., $b^{\prime} c^{\prime}, b a^{\prime}$ and $b a^{\prime} \vee a$ if $b \perp c$ and $a \leqq b$; if $b_{1} \leqq b_{2} \leqq \cdots$ then $\vee b_{i}=$ $b_{1} \vee b_{2} b_{1}^{\prime} \vee b_{3} b_{2}^{\prime} \vee \cdots$.

Consider the following three properties for $P$.

(W) $a \leqq b$ implies $b=b a^{\prime} \vee a$,

(W1) $a \leqq b$ and $b a^{\prime}=0$ imply $a=b$,

(W2) $a \leqq c$ and $b \perp c$ imply $(a \vee b) c=a .^{1}$

Lemma 1.1. If (W) holds then $a \perp b$ implies $b=(a \vee b) a^{\prime}$.

Proof. $a \leqq b^{\prime}$ so $b^{\prime}=b^{\prime} a^{\prime} \vee a$ by (W) and $b=\left(b^{\prime} a^{\prime} \vee a\right)^{\prime}=(a \vee b) a^{\prime}$

Lemma 1.2. If (W) holds and $a, b$ and $c$ are pairwise orthogonal, then $(a \vee b)(a \vee c)=a$ and $(a \vee b)(a \vee c)^{\prime}=b$.

Proof. $b \leqq a^{\prime}, b \leqq c^{\prime}$ imply $b \leqq a^{\prime} c^{\prime}$ so $a^{\prime} c^{\prime}=a^{\prime} c^{\prime} b^{\prime} \vee b$. Then $a=$ $a(a \vee c \vee b)=(a \vee b) b^{\prime}(a \vee c \vee b)$ by Lemma 1.1

$$
=(a \vee b)\left(a^{\prime} c^{\prime} b^{\prime} \vee b\right)^{\prime}=(a \vee b)\left(a^{\prime} c^{\prime}\right)^{\prime}=(a \vee b)(a \vee c) .
$$

${ }^{1}$ That is, $(a \vee b) c$ exists and is equal to $a$. In general, when $x$ exists a priori but $y$ may not, the assertion $y=x$ is understood to include the assertion that $y$ exists. 
Since $b=(a \vee b) a^{\prime}$ by Lemma 1.1 and $b \leqq c^{\prime}, \quad b=b c^{\prime}=(a \vee b) a^{\prime} c^{\prime}=$ $(a \vee b)(a \vee c)^{\prime}$.

LEMmA 1.3. (W), (W1) and (W2) are equivalent.

(W1) implies (W). Suppose $a \leqq b$. Then $a \vee b a^{\prime} \leqq b$ holds trivially and $b\left(a \vee b a^{\prime}\right)^{\prime}=b\left(a^{\prime}\left(b a^{\prime}\right)^{\prime}\right)=b a^{\prime}\left(b a^{\prime}\right)^{\prime}=0$ so $b=a \vee b a^{\prime} b v$ (W1).

(W) implies (W2). If $a \leqq c$ and $b \perp c$, then $c a^{\prime}, a$ and $b$ are orthogonal so $a=(a \vee b)\left(a \vee c a^{\prime}\right)$ Lemma 1.2 (since $(\mathrm{W})$ holds) $=(a \vee b) c$ by (W).

(W2) implies (W1). Suppose $a \leqq b$ and $b a^{\prime}=0$ Then $b \perp b^{\prime}$ so, by (W2), $a=\left(a \vee b^{\prime}\right) b=\left(b a^{\prime}\right)^{\prime} b=0^{\prime} b=1 b=b$.

$P$ is said to be weakly modular (relative to the given orthocomplementation) if it satisfies any and hence all of (W), (W1) and (W2). We assume now that $P$ is weakly modular and, borrowing a traditional term from the theory of probability, we call its members events.

Two events $a_{1}, a_{2}$ are said to commute or to be simultaneously measurable if there exist pairwise orthogonal events $b_{1}, b_{2}$ and $c$ such that $a_{i}=b_{i} \vee c$. The set of all events which commute with all other events is called the center $\mathscr{C}$ of $P$. If $\mathscr{C}=P, P$ is said to be commutative or deterministic. It is an easy consequence of Lemma 1.2 that $a$ and $b$ commute if and only if $a b, a b^{\prime}$ and $a^{\prime} b$ exist, $a=a b \vee a b^{\prime}$ and $b=a b \vee a^{\prime} b$, and hence that $P$ is deterministic if and only if it is a Boolean algebra.

Lemma 1.4. Suppose $a b$ and $a b^{\prime}$ exist and $a=a b \vee a b^{\prime}$. Then $a$ and $b$ commute.

Proof. $\quad a^{\prime}=\left(a b \vee a b^{\prime}\right)^{\prime}=(a b)^{\prime}\left(a b^{\prime}\right)^{\prime}=(a b)^{\prime}\left(b \vee a^{\prime}\right) \geqq(a b)^{\prime} b$ while $b \geqq(a b)^{\prime} b$ holds trivially. On the other hand, if $a^{\prime} \geqq c$ and $b \geqq c$ then $(a b)^{\prime} \geqq a^{\prime} \geqq c$ so $(a b)^{\prime} b \geqq c$. Hence $(a b)^{\prime} b=a^{\prime} b$ and so $b=(a b)^{\prime} b \vee a b=a^{\prime} b \vee a b$.

COROllary. If $a$ and $b$ commute, so do $a$ and $b^{\prime}$.

Proof. The statement of the lemma is symmetric in $b$ and $b^{\prime}$.

Lemma 1.5. Suppose $P$ is a lattice. Then $P$ is a Boolean algebra if and only if $a b=0$ implies $a \perp b$.

Proof. If $P$ is a Boolean algebra and $a b=0$, then $a 1=a=a\left(b \vee b^{\prime}\right)=$ $a b \vee a b^{\prime}=a b^{\prime}$ so $a \leqq b^{\prime}$. Conversely, for any $a$ and $b, a(a b)^{\prime} b=0$ so $a(a b)^{\prime} \leqq b^{\prime}$ by hypotheses. Then $a=a b \vee a(a b)^{\prime}=a b \vee a(a b)^{\prime} b^{\prime}=a b \vee a b^{\prime}$ since $b^{\prime} \leqq(a b)^{\prime}$.

If we interpret the weakly modular lattice $P$ as the logic of an abstract physical system, ${ }^{2} a \leqq b$ means " $a$ implies $b$ " and $a^{\prime}$ is the event

${ }^{2}$ Cf. [2]. 
"not $a$ ". If $a \perp b$, it is natural to say that $a$ and $b$ are "mutually exclusive" $-a$ implies not $b$ and $b$ implies not $a$-and in this case the question of the simultaneous occurrence of $a$ and $b$ is completely settled. If, however, $a b=0$ but $a$ and $b$ are not orthogonal, no experiment exists for the system whose outcome can indicate that $a$ and $b$ have both occurred even though $a$ and $b$ are not mutually exclusive. According to Lemma 1.5, the absence of this uncertainty is equivalent to the commutativity of $P$.

Digression. It may be shown that the notion of determinacy is further characterized in the following three ways (the statements depend on definitions which appear below). We suppose given a system of states and events $\mathscr{S}, P$.

(i) Let $X$ denote the real linear space of signed measures on $P$ generated by $\mathscr{S} . \quad P$ is deterministic if and only if $X$ is a pre- $L$-space in a certain natural sense (see [4]).

(ii) Define an observable, as in [7], to be a function $A$ from the Borel subsets of the real line $R$ to $P$ such that $A_{\phi}=0, A_{R}=1, A_{E} \perp A_{F}$ if $E \cap F=\phi$ and $A_{\cup_{E_{i}}}=\sum A_{E_{i}}$ if $E_{i} \cap E_{j}=\phi$ for $i \neq j ; A$ is bounded if $A_{E}=1$ for some bounded Borel set $E$. Given $x \in X$ (see (i) above) and a bounded observable $A$, let $\mu_{x ; A}$ denote the Borel measure on the line: $\mu_{x, A}(E)=x\left(A_{E}\right)$ and let $L_{A}$ denote the functional on $X$ : $L_{A}(x)=\int_{-\infty}^{\infty} \lambda d \mu_{x, A}(\lambda)$. The set $Y$ of all such $L_{A}$ is partially ordered as a subset of the dual of the partially ordered linear space $X . P$ is deteministic if and only if $Y$ is a lattice.

(iii) Suppose $P$ has a unit. Then $P$ is deterministic if and only if every pair $A, B$ of observables is simultaneously measurable in the following intuitive sense: there exist an observable $C$ and Borel functions $\alpha$ and $\beta$ from $R$ to $R$ (depending on $A$ and $B$ ) such that $A=\alpha(C)$ and $B=\beta(C)$ (where, by definition, $\alpha(C)_{E}=C_{\alpha^{-1}(E)}$ ).

A function $f$ from the weakly modular partially ordered set $P$ to the closed real unit interval is said to be a state for $P$ if $f(1)=1$ and $f$ is countably additive in the sense that whenever $\left\{a_{i}\right\}$ is orthogonal, $f\left(\mathrm{~V} a_{i}\right)=\sum f\left(a_{i}\right)$. It is easy to see that if $f$ is a state and $\left\{b_{i}\right\}$ is an increasing (decreasing) sequence of events with sup (inf) $b$, then $f\left(b_{i}\right) \rightarrow f(b)$.

Now suppose there exists a set $\mathscr{S}$ of states such that

(D) $a \leqq b$ if and only if $f(a) \leqq f(b)$ for all $f$ in $\mathscr{S}$.

Of course, if $a \leqq b$ and $f$ is any state, $f(a)=f(b)-f\left(b a^{\prime}\right) \leqq f(b)$.

We observe that

E1. If $f(a)=f(b)$ for all $f$ in $\mathscr{S}, a=b$,

E2. For each $a \in P$ there exists $b \in P$ such that $f(b)=1-f(a)$ for all $f$ in $\mathscr{S}$; there exists $c \in P$ such that $f(c)=0$ for all $f$ in $\mathscr{S}$,

E3. Let $\left\{a_{1}, a_{2}, \cdots\right\}$ be a sequence of elements of $P$ such that $i \neq j$ 
and $f \in \mathscr{S}$ imply $f\left(a_{i}\right)+f\left(a_{1}\right) \leqq 1$. Then there exists $a \in P$ such that $f(a)=\sum f\left(a_{i}\right)$ for all $f \in \mathscr{S}$.

Indeed, E1 is immediate from (D) and in E2 we need only set $b=a^{\prime}$, $c=0$. In E3, $f\left(a_{i}\right) \leqq 1-f\left(a_{j}\right)=f\left(a_{j}^{\prime}\right)$ implies $a_{i} \leqq a_{j}^{\prime}$ by (D) so the $\left\{a_{i}\right\}$ are mutually orthogonal and we may set $a=\mathrm{V} a_{i}$.

Suppose, on the other hand, that we are given a set $P$ (without any a priori structure) and a set $\mathscr{S}$ of functions from $P$ to the closed unit interval satisfying E1-E3. The elements $b$ and $c$ of E2 are unique by E1 and are denoted $a^{\prime}$ and 0 respectively and we let $1=0^{\prime}$; the element $a$ of E3 is also unique by E1 and is denoted $\sum a_{i}$. Let a partial ordering be defined in $P$ by (D); evidently 0 and 1 are the least and greatest elements of $P$ and $a \rightarrow a^{\prime}$ is an orthocomplementation. We shall show that the orthocomplemented partly ordered set $P$ is weakly modular and $\mathscr{S}$ is a collection of states for $P$ (which trivially satisfies (D)).

Let $\left\{a_{i}\right\}$ be orthogonal, let $I_{1}, I_{2}, \cdots$ be a partition of the positive integers and let $b_{i}=\sum_{j \in I_{i}} a$, where $\sum$ denotes the sum of the $a_{j}$ in the sense of E3 . It follows at once from the fact that the sum of a convergent series of nonnegative numbers is unaffected by a rearrange ment of its terms that the $b_{i}$ are pairwise orthogonal and $\sum b_{i}=\sum a_{i}$. As a particular case we have,

LEMMA 1.6. If $a_{1}, a_{2}, \cdots$ are pairwise orthogonal and $b \perp a_{i}$ for every $i$, then $b \perp \sum a_{i}$.

LEMmA 1.7. If $a_{1}, a_{2}, \cdots$ are pairwise orthogonal, then $\Sigma a_{i}=\bigvee a_{i}$.

Proof. Clearly $a=\sum a_{i} \geqq a_{j}$ for all $j$. If $b \geqq a$, for all $j, a_{j} \perp b^{\prime}$ so $a \perp b^{\prime}$ by Lemma 1.6 ; i.e., $b \geqq a$.

Now suppose $a \leqq b$. Then $a \perp b^{\prime}$ so $a+b^{\prime}$ exists by E3 and equals $a \vee b^{\prime}$ by Lemma 1.7; hence $b a^{\prime}=\left(a \vee b^{\prime}\right)^{\prime}$ exists. Since $b a^{\prime} \perp a, b a^{\prime} \vee a$ exists by E3 and Lemma 1.7. Then if $f \in \mathscr{S}$,

$$
\begin{aligned}
f\left(b a^{\prime} \vee a\right) & =f\left(b a^{\prime}\right)+f(a)=1-f\left(\left(b a^{\prime}\right)^{\prime}\right)+f(a)=1-f\left(b^{\prime} \vee a\right)+f(a) \\
& =1-f\left(b^{\prime}\right)-f(a)+f(a)=1-f\left(b^{\prime}\right)=f(b)
\end{aligned}
$$

and it follows from E1 that $b=b a^{\prime} \vee a$, i.e., (W) holds and $P$ is weakly modular. If $\left\{a_{i}\right\}$ is orthogonal, $f\left(\bigvee a_{i}\right)=f\left(\sum a_{i}\right)$ by Lemma $1.7=\sum f\left(a_{i}\right)$ and so $f$ is a state for $P$.

Let $P$ be a weakly modular partially ordered set and let $\mathscr{S}$ be a family of states for $P$ which determines the order relation in $P$ (as in (D)). The pair $\mathscr{S}, P$ will be called a system of state and events, or simply a system, if it has the following five properties.

E4. (Axiom of separability) Every orthogonal subset of $P$ contains at 
most countably many non-zero elements.

E5. $P$ is a lattice.

S1. $\mathscr{S}$ is closed under countable convex combination; i.e., if $f_{1}, f_{2}, \ldots$ are in $\mathscr{S}$ and $\lambda_{1}, \lambda_{2}, \cdots$ are nonnegative real numbers with $\sum \lambda_{i}=1$, then $\sum \lambda_{i} f_{i} \in \mathscr{S}$.

S2. If $a$ is a non-zero event, there exists $f \in \mathscr{f}$ such that $f(a)=1$. S3. If $f(a)=0$ and $f(b)=0$, then $f(a \vee b)=0$.

The following series of lemmas, culminating in Theorem 1.1, develop a number of regularity properties that systems enjoy; interrelations among the properties are exhibited in accompanying remarks.

LemMa 1.8. Suppose $P$ is separable (i.e., satisfies E4). Then if $Q$ is a nonempty chain in $P, a$ sequence $\left\{a_{1}, a_{2}, \cdots\right\}$ of elements of $Q$ may be found such that $\vee a_{i}=\sup Q ;$ in particular, $\sup Q$ exists in $P$.

Proof. Let $Q$ be a nonempty chain in $P$ and let $T$ be the set of all events of the form $a b^{\prime}$ where $a \in Q$ and $b<a$. Let $\left\{c_{i}\right\}$ be a maximal set of pairwise orthogonal non-zero elements of $T$ which exists by Zorn's lemma and is countable by E4. Say $c_{i}=a_{i} b_{i}^{\prime}$ where $a_{i} \in Q, a_{1}<a_{2}<\cdots$ and $b_{i}<a_{i}$ and let $a=\bigvee a_{i}$. Suppose there exists $b \in Q$ such that $b \geqq a$. Then since $b \nless a_{i}, a_{i}<b$ holds for all $i$ since $Q$ is a chain. Hence $a<b$ and the non-zero event $b a^{\prime}$ belongs to $T$ and is orthogonal to all the $c_{i}$ contrary to the maximality of $\left\{c_{i}\right\}$.

A cut in $P$ is a subset of $P$ which contains all lower bounds of the set of its upper bounds. If $Q \subseteq P$, we denote by $\bar{Q}$ the smallest cut containing $Q$. Thus, for $a \in P, \bar{a}=\{b \in P: b \leqq a\}$ and for $Q \subseteq P$, $\bar{Q}=\cap \bar{a}: Q \subseteq \bar{a}$. The mapping $Q \rightarrow \bar{Q}$ is evidently a closure operation in the power class $\mathscr{B}(P)$ of $P$ (see [1]); hence the set $\bar{P}$ of all cuts in $P$ is a complete lattice under inclusion.

Lemma 1.9. If $P$ is a lattice and every chain in $P$ has a sup in $P$, then $P$ is a complete lattice.

REMARK. If $P$ is a lattice and $\left\{b_{i}\right\} \subseteq P, \bigvee b_{i}=\mathrm{V}\left(b_{1} \vee \cdots \vee b_{i}\right)$, i.e., $P$ is $\sigma$-complete.

Proof. Suppose $Q \subseteq P$, let $Q_{1}$ be a chain in $\bar{Q}$ and let $b=\sup Q_{1}$. If $a \in P$ such that $Q \leqq \bar{a}$, then $Q_{1} \leqq \bar{a}$ so $b \leqq a$, i.e., $b \in \bar{Q}$. It follows now from Zorn's lemma that $\bar{Q}$ contains a maximal element $b$. The assumption that $P$ is a lattice clearly implies that $\bar{Q}$ is a sublattice of $P$ and so if $a \in Q, a \vee b \in \bar{Q}$. Then by the maximality of $b$ in $\bar{Q}$, $a \vee b=b$ so $a \leqq b$; thus, $Q \subseteq \bar{b}$ and $b=\sup Q$. Dually, $\inf Q=\left(\sup a^{\prime}: a \in Q\right)^{\prime}$.

For $Q \subseteq P$ let $Q^{\circ}=\{f \in \mathscr{S}: f(a)=0$ for all $a \in Q\}$ and if $T \subseteq \mathscr{S}$ let $T^{\circ}=\{a \in \mathscr{S}: f(a)=0$ for all $f \in T\}$. Clearly $Q=Q^{\circ \circ}$ and if $Q_{1} \subseteq Q_{2}$, 
then $Q_{2}^{\circ} \subseteq Q_{1}^{\circ}$, and similarly for the subsets of $\mathscr{S}$. The first relation implies $Q^{\circ} \cong Q^{\circ \circ \circ}$ and the second applied to the first yields $Q^{\circ \circ \circ} \cong Q^{\circ}$; thus $Q^{\circ}=Q^{\circ \circ \circ}$ and similarly, $T^{\circ}=T^{\circ 0 \circ}$. A subset $H$ of $P$ or of $\mathscr{S}$ such that $H=H^{\circ \circ}$ is called an annihilator, and the mapping $H \rightarrow H^{\circ}$ is a one-to-one inclusion inverting correspondence between the annihilators in $\mathscr{B}(P)$ and those in $\mathscr{B}(\mathscr{S})$. In this notation, S3 is: $a^{\circ} \cap b^{\circ}=(a \vee b)^{\circ}$. It is easy to see that if $\mathscr{S}, P$ has any one of the following three properties, it has the others.

(4) $a^{\circ \circ} \cong b^{\circ \circ}$ implies $a \leqq b$,

(5) $b^{\circ} \subseteq a^{\circ}$ implies $a \leqq b$,

(6) if $f(a)=1$ whenever $f(b)=1$, then $b \leqq a$.

Lemma 1.10. If E5, S2 and S3 hold for $\mathscr{S}, P$, so do (4)-(6).

Proof. Suppose $b \circ \cong a^{\circ}$. Then $b^{\circ}=b^{\circ} \cap a^{\circ}=(a \vee b)^{\circ}$ by S3. If $a \vee b \neq b$, then $(a \vee b) b^{\prime} \neq 0$ by (W1) so, by S2, there exists $f \in \mathscr{S}$ such that $f\left((a \vee b) b^{\prime}\right)=1$. Then $f \in b^{\circ}$ so $f \in a^{\circ}$ and $f(a \vee b)=0$ by S3. But $f(a \vee b) \geqq f\left((a \vee b) b^{\prime}\right)=1$, so $a \vee b=b, a \leqq b$ must hold.

Lemma 1.11. Suppose $P$ is a separable lattice. Then $P$ is a complete lattice and $Q \subseteq P$ implies there exists $Q_{1} \leqq Q$ with at most countably many elements such that $\sup Q_{1}=\sup Q$.

Proof. $P$ is a complete lattice by Lemmas 1.8 and 1.9 . Let $Q$ be a nonempty subset of $P$ and let $a=\sup Q$. Let $T$ denote the set of all joins of countable subsets of $Q$. If $T_{1}$ is a chain in $T$, its join is obtainable as the join of a countable subsets of $T_{1}$ by Lemma 1.8 and hence belongs to $T$. Hence, we may use Zorn's lemma to extract a maximal element $a$ from $T$, and then, clearly, $a=\sup Q$.

REmark. The converse is also true. Indeed, suppose $\left\{a_{\alpha}\right\}$ is orthogonal and $a$ is its join; by hypothesis $a=\mathrm{V} a_{\alpha_{i}}$ for appropriate $\alpha_{i}$. If $\alpha \notin\left\{\alpha_{i}\right\}$, then $a_{\alpha} \perp a$ by Lemma 1.6. Since $a_{\alpha} \leqq a$ by definition of $a, a_{a}=0$.

We consider now the general form of S3:

(7) If $a$ is the sup of the subset $Q$ of $P$ and $f(b)=0$ for all $b \in Q$, then $f(a)=0$ (equivalently: $Q^{\circ}=a^{\circ}$ ).

It is easy to see that if E5 and S3 hold, so does (7) whenever $Q$ has countably many elements.

Lemma 1.12. If E4, E5 and S3 hold for $\mathscr{S}, P$, so does (7).

Proof. Let $Q \cong P, a=\sup Q$ and let $f \in Q^{\circ}$. By Lemma 1.11 we may choose a sequence $\left\{a_{\imath}\right\} \subseteq Q$ such that $a=\bigvee a_{i}$; let $b_{n}=a_{1} \vee \cdots \vee a_{n}$. 
Then $b_{1} \leqq b_{2} \leqq \cdots, f\left(b_{i}\right)=0$ imply $f\left(\mathrm{~V} b_{i}\right)=f(a)=0$.

An event $a$ is said to be a carrier of the state $f$ on $P$ if $f(b)=0$ is equivalent to $b \perp a$; if a carrier exists for $f$ it is clearly unique and is denoted $a_{f}$. Evidently, if $f$ is a state with a carrier, $f(b)=1$ if and only if $a_{f} \leqq b$, and $f^{\circ}=a_{f}^{\prime \circ 0}=\overline{a_{f}^{\prime}}$.

Lemma 1.13. Suppose $P$ is a complete lattice. Then if $\mathscr{S}, P$ satisfies (7), it also satisfies

(8) Every $f \in \mathscr{S}$ has a carrier in $P$.

Proof. $a_{f}=\left(\sup f^{\circ}\right)^{\prime}$.

Remark 1. Conversely, if $P$ is a complete lattice and $\mathscr{S}, P$ satisfies (8), then (7) holds. Indeed, if $Q \subseteq P$ and $a=\sup Q$, let $f \in Q^{\circ}$. Then $b \leqq a_{f}^{\prime}$ for all $b \in Q$ so $a \leqq a_{f}^{\prime}$ and hence $f \in a^{\circ}$, i.e., $Q^{\circ} \cong a^{\circ}$.

REMARK 2. (8) is equivalent to the following: $Q \subseteq P, f \in Q^{\circ}, f(a)=1$ imply there exists $b \leqq a$ such that $f(b)=1$ and $b \perp Q$. For if (8) holds, we may take $b=a_{f}$ while, conversely, given $f$, observe that since $f(1)=1$, the hypothesis implies the existence of $b$ such that $f(b)=1$ and $b \perp f^{\circ}$; clearly $b=a_{f}$.

Lemma 1.14. Suppose $\mathscr{S}, P$ satisfies (4)-(6) and (8). Then it also satisfies

(9) $\bar{Q}=Q^{\circ \circ}$ for every subset $Q$ of $P$.

Proof. $Q^{\circ \circ}=\bigcap \overline{a_{f}^{\prime}}: f \in Q^{\circ}$ by definition and (8). But $Q \subseteq \overline{a_{f}^{\prime}}$ for all $f \in Q^{\circ}$ so $\bar{Q}=\{\cap \bar{a}: Q \subseteq \bar{a}) \leqq\left(\cap \overline{a_{f}^{\prime}}: f \in Q^{\circ}\right\}=Q^{\circ \circ}$. On the other hand, $b \in Q^{\circ \circ}$ implies $Q^{\circ}=Q^{\circ \circ \circ} \subseteq b^{\circ}$ while $Q \subseteq \bar{a}$ implies $a^{\circ} \cong Q^{\circ}$. Hence $a^{\circ} \cong b^{\circ}$ so $b \leqq a$ by (5). Thus, $Q^{\circ \circ} \cong \bar{Q}$ so $Q^{\circ \circ}=\bar{Q}$.

Remark. If $\mathscr{S}, P$ satisfies (9), it also satisfies (4)-(6) and (7). Indeed, (4)-(6) are immediate. To prove (7), suppose $a \in P$ is the sup of the subset $Q$ of $P$. Then $\bar{a}=\bar{Q}=Q^{\circ \circ}$ by (9) so $Q^{\circ \circ \circ}=Q^{\circ}=a^{\circ}$.

Lemma 1.15. Suppose $\mathscr{S}, P$ satisfies E4, E5, S1, S2 and (8). Then (10) Every non-zero event is the carrier of some $f \in \mathscr{S}$.

Proof. We may use the conclusion of Lemma 1.11. Assuming $a \neq 0$, it follows from S2 that $a^{\prime \circ} \neq \phi$; let $b=\bigvee a_{f}: f \in a^{\prime \circ}$. Since $a_{f} \leqq a$ for all $f \in a^{\prime \circ}, b \leqq a$. If $a b^{\prime} \neq 0$, choose $g \in \mathscr{S}$ with $g\left(a b^{\prime}\right)=1$; then $0=g\left(\left(a b^{\prime}\right)^{\prime}\right)=g\left(a^{\prime} \vee b\right) \geqq g\left(a^{\prime}\right)$ so $g \in a^{\prime \circ}$ and $a_{g} \leqq b$ by definition of $b$. On the other hand, $g\left(b^{\prime}\right) \geqq g\left(a b^{\prime}\right)=1$ implies $a_{g} \leqq b^{\prime}$ so $a_{g}=0$. Since 0 cannot be the carrier of a state, $a b^{\prime}=0$ must hold and so $a=b$ by (W1). Choose $\left\{f_{1}, f_{2}, \cdots\right\} \subseteq a^{\prime \circ}$ such that $a=\mathrm{V} a_{f_{i}} ; f_{0}=f_{1} / 2+f_{2} / 2^{2}+\cdots$ belongs 
to $\mathscr{S}$ by S1. Then $f_{0}(a)=1$ so $a_{f_{0}} \leqq a$; but, clearly, $f_{i}\left(a_{f_{0}}\right)=1$ so $a_{f_{i}} \leqq a_{f_{0}}$ for $i=1,2, \cdots$ and $a=a_{f_{i}} \leqq a_{\rho_{0}}$. Hence $a=a_{f_{0}}$ and the proof is complete.

Remark. If $\mathscr{S}, P$ satisfies (10), it also satisfies (4)-(6), for suppose $a^{\circ \circ} \subseteq b^{\circ \circ}$. Now $a \leqq b$ holds trivially if $b=1$ so suppose $b \neq 1$ and choose $f \in \mathscr{S}$ in accordance with (10) such that $a_{f}=b^{\prime}$. Then $\bar{a} \cong a^{\circ \circ} \leqq$ $b^{\circ \circ}=a_{f}^{\prime \circ \circ}=\overline{a_{f}^{\prime}}=\bar{b}$ so $a \leqq b$.

A state $f$ on $P$ such that $f(a)=0$ implies $a=0$ is said to be a unit for $P$. It is easy to see that if $P$ has a unit, it is separable.

Lemma 1.16. If $\mathscr{S}, P$ satisfies (10), $\mathscr{S}$ contains a unit.

Proof. $f \in \mathscr{S}$ such that $a_{f}=1$ is a unit.

We have proved, in particular:

Theorem 1.1. Let $\mathscr{S}, P$ be a system of states and events. Then $P$ is a complete lattice and the sup of any infinite family of its elements is obtainable as the sup of a countable subfamily. Furthermore, $\mathscr{S}$ contains a unit for $P$, and the pair $\mathscr{S}, P$ has the following properties.

(6) If $f(a)=1$ whenever $f(b)=1$, then $b \leqq a$.

(7) If $Q \subseteq P$ and $f(b)=0$ for all $b \in Q$, then $f(\sup Q)=0$.

(8) Every $f \in \mathscr{S}$ has a carrier in $P$.

(9) $\bar{Q}=Q^{\circ \circ}$ for every $Q \subseteq P$.

(10) Every non-zero event is the carrier of some $f \in \mathscr{S}$.

2. The model for non-relativistic quantum mechanics. We shall show that certain further constraints on a system $\mathscr{S}, P$ imply that $P$ is isomorphic to the lattice of closed subspaces of a separable infinite dimensional Hilbert space.

We recall that $a$ covers $b$ means that $a>b$ and $a \geqq c>b$ implies $a=c$. A point is an element which covers 0 and $P$ is atomic if each of its elements is the join of points. We shall call an event finite if it is the join of a finite number of points and let $P_{f}$ denote the set of all finite events. Suppose now that $\mathscr{S}, P$ is a system satisfying

(A). $P$ is atomic; $1 \notin P_{f}$.

Let $(a)$ denote the lattice under $a$; clearly $(a)$ is weakly modular relative to the orthocomplementation $b \rightarrow a b^{\prime}$. We assume

(M). Let $a \in P_{f}$ and suppose $b, c$ and $d$ are elements of $(a)$ with $d \leqq c$ and $b c=0$. Then $(d \vee b) c=d .^{3}$

\footnotetext{
${ }^{3}$ If $d \leqq c$ and $b \perp c,(d \vee b) c=d$ by weak modularity (cf. Lemma 1.3); thus, (M) asserts that, under finite elements, $b c=0$ bears a certain resemblance to $b \perp c$.
} 
Lemma 2.1. If $a$ is finite, (a) is modular.

Proof. Let $d, b$ and $c$ be elements of $(a)$ with $d \leqq c$. Then $d \vee b c \leqq c$ and $b(b c)^{\prime} c=0$ so writing $b=b c \vee b(b c)^{\prime}$ (by weak modularity) and letting $d \vee b c, b(b c)^{\prime}$ and $c$ play the roles of $d, b$ and $c$ of (M) respectively in the last of the following equalities, $(d \vee b) c=(d \vee(b c \vee$ $\left.\left.b(b c)^{\prime}\right)\right) c=\left((d \vee b c) \vee b(b c)^{\prime}\right) c=d \vee b c$.

REMARK. This result is valid for an arbitrary orthocomplemented lattice $L$; that is, if $L$ has the property attributed to $(a)$ in $(M)$, it obviously satisfies (W2) of $\S 1$, hence is weakly modular (see Lemma 1.3), so the proof applies, and $L$ is modular.

Lemma 2.2. Suppse $a>b$. Then a covers $b$ if and only if $a b^{\prime}$ is a point.

Proof. Suppose $a b^{\prime}$ is a point and $a \geqq c>b$. Then $0<c b^{\prime}$ by (W1) so $c b^{\prime} \leqq a b^{\prime}$ implies $c b^{\prime}=a b^{\prime}$. Hence $c=c b^{\prime} \vee b=a b^{\prime} \vee b=a$, i.e., $a$ covers $b$. If $a b^{\prime}$ is not a point, $a b^{\prime}>c>0$ for some $c \in P$ and then $a=b \vee a b^{\prime}=b \vee a b^{\prime} c^{\prime} \vee c>b \vee c>b$ so $a$ does not cover $b$.

Corollary. Let $a \in P$. The chain $0=a_{0}<a_{1}<a_{2}<\cdots$ is maximal in (a) if and only if $a_{i} a_{i-1}^{\prime}$ is a point for $i=1,2, \cdots$ and $\vee a_{i}=a$.

Lemma 2.3. ([1, pp. 66,67]) Let $a \in P_{f}$ and suppose every orthogonal set of points in $(a)$ is finite. Then if $b \leqq a$ and $\left\{a_{1}, \cdots, a_{n}\right\}$ and $\left\{b_{1}, \cdots, b_{m}\right\}$ are two maximal orthogonal sets of points in $(b), m=n$.

LEMmA 2.4. ([1, p. 66]) Let $a \in P_{f}$ and suppose $b, c$ and $d$ are elements of $(a)$ such that $b$ covers $d, b$ and $c$ are not comparable and $d<c$. Then $b \vee c$ covers $c$.

For $a \in P_{f}$ let $\operatorname{dim} a=-1+\min \{n: a$ is the join of $n$ points $\}$ and let $P_{i}=\left\{a \in P_{f}: \operatorname{dim} a=i\right\}, i=-1,0,1, \cdots$. Clearly, $P_{-1}=\{0\}, P_{0}$ is the set of points and $P_{f}=\bigcup P_{i}$.

Suppose there exists $\alpha \in P_{f}$ such that $(\alpha)$ contains an infinite orthogonal set $\left\{b_{i}\right\}_{i=0}^{\infty}$ of points, and assume that $n=\operatorname{dim} a$ is a minimum for $a$ with this property; clearly $n>0$. Let $a_{0}, \cdots, a_{n}$ be points with join $a$. Since $\operatorname{dim} a_{0} \vee \cdots \vee a_{n-1}=n-1$, Lemma 2.3 implies the existence of orthogonal points $c_{0}, \cdots, c_{n-1}$ such that $c_{0} \vee \cdots \vee c_{n-1}=a_{0} \vee \cdots \vee a_{n-1}$. Then $a_{n}$ covers 0 and is not comparable with $a_{0} \vee \cdots \vee a_{n-1}$ so $a=$ $a_{0} \vee \cdots \vee a_{n-1} \vee a_{n}$ covers $a_{0} \vee \cdots \vee a_{n-1}$ by Lemma 2.4 and hence $c_{n}=a\left(a_{0} \vee \cdots \vee a_{n-1}\right)^{\prime}$ is a point by Lemma 2.2 ; clearly $a=c_{0} \vee \cdots \vee c_{n}$ and the $c_{i}$ are orthogonal. Now $c_{0} \neq b_{0}$, for otherwise $c_{1} \vee \cdots \vee c_{n}=$ $b_{1} \vee b_{2} \vee \cdots$ contrary to the choice of $a$ with minimum dimension. Hence 
$c_{0} \vee b_{0}$ covers $c_{0}$, so $d_{0}=\left(c_{0} \vee b_{0}\right) c_{0}^{\prime}$ is a point. For $i=1,2, \cdots$ let $d_{i}=\left(c_{0} \vee b_{0} \vee \cdots \vee b_{i}\right)\left(c_{0} \vee b_{0} \vee \cdots \vee b_{i-1}\right)^{\prime}$. If $b_{i} \leqq c_{0} \vee b_{0} \vee \cdots \vee b_{i-1}$, $d_{i}=0$ while if not, $c_{0} \vee b_{0} \vee \cdots \vee b_{i}$ covers $c_{0} \vee b_{0} \vee \cdots \vee b_{i-1}$ by Lemma 2.4 so $d_{i}$ is a point. Since all the $d_{i}$ are orthogonal and lie under $a c_{0}^{\prime}$, all but a finite number must be 0 , since $\operatorname{dim} a c_{0}^{\prime}=n-1<\operatorname{dim} \alpha$. Since $\mathrm{V} d_{i}=a c_{0}^{\prime}$, exactly $n$ of the $d_{i}$ are points by Lemma 2.3 and we assume without essential loss of generality that $d_{0}, \cdots, d_{n-1}$ are points. But then $a=a c_{0}^{\prime} \vee c_{0}=d_{0} \vee \cdots \vee d_{n-1} \vee c_{0}=b_{0} \vee \cdots \vee b_{n-1} \vee c_{0}$. Since $c_{0}$ is a point not comparable with $b_{0} \vee \cdots \vee b_{n-1}$, a covers $b_{0} \vee \cdots \vee b_{n-1}$ and so $e=a\left(b_{0} \cdots b_{n-1}\right)^{\prime}$ is a point. But $b_{i} \leqq e$ for $i \geqq n$ and so all but one of these $b_{i}$ must be zero. This contradiction completes the proof of

LEMMA 2.5. If a is finite, every orthogonal set of points in (a) is finite.

COROLlaRY. If $a$ is finite and $\left\{a_{\imath}\right\}_{\imath=0}^{n}$ is an orthogonal set of points in (a) with join a then $n=\operatorname{dim} a$.

We call the elements of $P_{1}$ lines, of $P_{2}$, planes, and use the following notation: if $a \in P,(a)_{i}=\{b \leqq a: \operatorname{dim} b=i\}, i=-1,0,1, \cdots$.

We make the following assumption of homogeneity:

(H) If $a$ and $b$ are finite elements of the same dimension, then $(a)$ and (b) are isomorphic.

Lemma 2.6. Suppose $P$ contains a pair of distinct points $a_{0}, b_{0}$ such that the line $a_{0} \vee b_{0}$ contains no third point. Then $P$ is deterministic.

Proof. $\left(a_{0} \vee b_{0}\right) b_{0}^{\prime}$ is a point distinct from $b_{0}$ so is equal to $a_{0}$ by hypothesis and hence $a_{0} \perp b_{0}$. It follows now from $(\mathrm{H})$ that if $a_{1}$ and $b_{1}$ are any two distinct points, then $a_{1} \perp b_{1}$. Hence if $a$ and $b$ are events with $a b=0, a=\bigvee a_{1}: a_{1} \in(a)_{0} \leqq \bigwedge b_{1}^{\prime}: b_{1} \in(b)_{0}=\left(\bigvee b_{1}: b_{1} \in(b)_{0}\right)^{\prime}=b^{\prime}$ so $a \perp b$ and $P$ is deterministic by Lemma 1.5 .

We assume

(ND) $P$ is not deterministic.

COROLLARY. Every line contains at least three distinct points.

Lemma 2.7. $\mathscr{C}=\{0,1\}$.

Proof. Suppose $a \in \mathscr{C}$ with $0<a<1$. Then there exist points $b_{1}$ and $b_{2}$ such that $b_{1} \leqq \alpha$ and $b_{2} \leqq a^{\prime}$. Let $c$ be a point in $b_{1} \vee b_{2}$ distinct from $b_{1}$ and $b_{2}$. Then $c=c a \vee c a^{\prime}$ so either $c \leqq \alpha$ or $c \leqq \alpha^{\prime}$ since $c$ is a point. But the former implies that $b_{2}<a$ since then $b_{1} \vee b_{2}=b_{1} \vee c \leqq a$ 
and similarly the latter implies that $b_{1}<a^{\prime}$; hence the assumption $0<$ $a<1$ is untenable.

We have shown that for $a \in P_{\rho},(a)$ is an orthocomplemented, modular lattice of finite dimension with trivial center and at least three points on each line. Thus, we have (see e.g., [1, Theorem 6, p. 120]):

THEOREM 2.1. Let $\mathscr{S}, P$ be a system satisfying (A), (M), (H) and (ND). Then the lattice under every finite element of $P$ is a projective geometry.

It follows from $(\mathrm{H})$ that there exists a division ring $D$ such that $a$ coordinatizing division ring $^{4}$ for any finite $(a)$ is isomorphic to $D$. We shall make use of the natural metric $\rho$ for $P: \rho(a, b)=\sup |f(a)-f(b)|: f \in \mathscr{S}$.

Lemma 2.8. Orthocomplementation is continuous in (a) for any $a \in P$. That is, if $\left\{b_{n}\right\} \subset(a), b \in(a)$ and $b_{n} \rightarrow b$, then $a b_{n}^{\prime} \rightarrow a b^{\prime}$.

Proof. Given $\varepsilon>0$ choose $N$ so that $n>N$ implies that $\rho\left(b_{n}, b\right)<\varepsilon$. Then if $f \in \mathscr{S}$ and $n>N$,

$$
\begin{aligned}
\varepsilon>\mid & f\left(b_{n}\right)-f(b)|=|(1-f(b))-\left(1-f\left(b_{n}\right)\right) \mid \\
& =\left|f\left(b^{\prime}\right)-f\left(b_{n}^{\prime}\right)\right|=\left|f\left(b^{\prime} a \vee a^{\prime}\right)-f\left(b_{n}^{\prime} a \vee a^{\prime}\right)\right| \\
& =\left|f\left(b^{\prime} a\right)+f\left(a^{\prime}\right)-f\left(b_{n}^{\prime} a\right)-f\left(a^{\prime}\right)\right|=\left|f\left(b^{\prime} a\right)-f\left(b_{n}^{\prime} a\right)\right| .
\end{aligned}
$$

Thus, $\rho\left(a b_{n}^{\prime}, a b^{\prime}\right)<\varepsilon$ and the result follows.

We assume now

$\left(\mathrm{C}^{\prime}\right)$ If $a$ is finite and $0 \leqq i \leqq \operatorname{dim} a,(a)_{i}$ is compact.

Remark. It seems reasonable to suppose that there exists $\varepsilon>0$ so small that if the probabilities of occurrence of two events $b$ and $c$ differ in every state by less than $\varepsilon$, then $b=c$, i.e., $b$ and $c$ are operationally identical. The completeness of $(a)_{i}$ is clearly weaker than this operational assumption. The assumption that $(a)_{i}$, in addition to being complete, is totally bounded, may be paraphrased as follows: for each $\varepsilon>0$ there exists a finite set $\left\{b_{1}, \cdots, b_{m}\right\}$ of elements of $(a)_{i}$ such that given any $b$ in $(a)_{i}$ and $f \in \mathscr{S}$ the probability of occurrence of the event $b$ in the state $f$ differs from the probability of occurrence of one of the $b$, in $f$ by an amount less than $\varepsilon$.

Lemma 2.9. Let a be a finite event of dimension at least two. Let $0 \leqq i, j<\operatorname{dim} a$, let $\left\{b_{n}\right\} \subset(a), \quad\left\{c_{n}\right\} \subset(a)$ with $\operatorname{dim} b_{n}=i$ and $\operatorname{dim} c_{n}=j$ for all $n$. Suppose that $b_{n} \rightarrow b$ and $c_{n} \rightarrow c$ where $b$ and $c$ are in "general position," i.e., $\operatorname{dim} b \vee c=\min (\operatorname{dim} a, i+j+1)$. Then $b_{n} \vee c_{n} \rightarrow b \vee c$ and, dually, $b_{n} c_{n} \rightarrow b c$.

\footnotetext{
${ }^{4}[\mathbf{1}$, Theorem 15, p. 131].
} 
Proof. $\left\{b_{n} \vee c_{n}\right\}$ clusters at some $d \leqq a$ by $\left(\mathrm{C}^{\prime}\right)$; assume for convenience that $b_{n} \vee c_{n} \rightarrow d$. Let $\varepsilon>0$ and choose $N$ so that $n>N$ implies $\rho\left(b_{n}, b\right)<\varepsilon / 2$ and $\rho\left(b_{n} \vee c_{n}, d\right)<\varepsilon / 2$. Then if $f(b)=1$ and $n>N$, $f(d)+\varepsilon / 2>f\left(b_{n} \vee c_{n}\right) \geqq f\left(b_{n}\right)>1-\varepsilon / 2$ so $f(d)>1-\varepsilon$. Hence $f(d)=1$ and so $b \leqq d$ by (6) of Theorem 1.1. Similarly $c \leqq d$ so $b \vee c \leqq d$. Since $\operatorname{dim} d \leqq \max _{n} \operatorname{dim} b_{n} \vee c_{n} \operatorname{dim} b \vee c, b \vee c=d$ must hold. The dual follows from Lemma 2.8 .

Corollary. Let $a \in P_{f}$. Then, in $(a)$, the lattice operations are continuous in both variables simultaneously.

We have therefore

LEMma $2.10 .^{5} \quad D$ is a locally compact division ring.

We now assume

(Co) For some $b \in P_{f}$ and real interval $I$ there exists a continuous nonconstant function $t \rightarrow a_{t}$ from $I$ to $(b)$.

Remark. Postulate (Co) may be obtained from the following "intuitive" assumptions. There exist a one-parameter family $L_{t}$ of mappings of $\mathscr{S}$ on $\mathscr{S}$ (describing how the states change with time (regarded as a real parameter)-corresponding to certain assumptions concerning the dynamics of the system (see $[6,7])$ ) and a state $f$ such that, letting $a_{t}$ denote the carrier of $L_{t}(f), a_{t}$ is continuous, non-constant and remains in some finite $(b)$ for all $t$ in an interval $I$.

For convenience assume $I=[0,1]$, let $n=\operatorname{dim} b, m=\operatorname{dim} a_{0}$. It follows at once from the continuity of $\alpha_{t}$ and the compactness of $(b)_{m}$ that $\operatorname{dim} a_{t}=m$ for all $\mathrm{t} \in I$. Suppose $m>0$. Without essential loss of generality we assume that $a_{t} \neq a_{0}$ for $t>0$ and choose a point $c<a_{0}$ such that $c \$ a_{t}$ for (again, for convenience) $t>0$. Let $d=c \vee a_{0}^{\prime}$. Choose $\delta>0$ such that $0 \leqq t<\delta$ implies $\rho\left(a_{0}, a_{t}\right)<1 / 2$. Then for such $t$, $a_{t} a_{0}^{\prime}=0$, for otherwise there exists $f \in \mathscr{S}$ such that $f\left(a_{t} a_{0}^{\prime}\right)=1$ so $f\left(a_{t}\right)=f\left(a_{0}^{\prime}\right)=1$. But then $\left|f\left(a_{0}\right)-f\left(a_{t}\right)\right|=\left|f\left(a_{0}\right)-1\right|<1 / 2$ implies $f\left(a_{0}\right)>1 / 2$, a contradiction. Hence, taking $\delta=1$ for convenience, $d a_{t}=d_{t}$ is a point for all $t$ (for $d_{t} \neq 0$ by a count of dimension while $a_{0}^{\prime} a_{t}=0$ implies $\left.\operatorname{dim} d a_{t} \leqq 0\right)$. Since $d_{0}=c$ and $d_{t} \neq c$ for $t>0, d_{t}$ is not constant, while it follows from Lemma 2.9 that $d_{t}$ is a continuous function of $t$; in case $m=0$ we set $d_{t}=a_{t}$. Again by continuity and without essential loss of generality, we can find a point $e^{(1)}$ disjoint from $\left\{d_{t}\right\}_{t \in I}$ and hyperplane $h^{(1)}$ such that $\left(e^{(1)} \vee d_{t}\right) h^{(1)}=d_{t}^{(1)}$, which is automatically continuous, is not constant. Similarly, if $\operatorname{dim} h^{(1)}=n-1>1$, we can find $e^{(2)} \varepsilon h^{(1)}$ disjoint from $\left\{d_{t}^{(1)}\right\}$ and $h^{(2)}=h^{(1)}$ with $\operatorname{dim} h^{(2)}=n-2$ such that $d_{t}^{(2)}=\left(e^{(2)} \vee d_{t}^{(1)}\right) h^{(2)}$ is non-constant in some subinterval of $I$. Continuing in this way, we arrive finally at a continuous non-constant function

${ }^{5}$ See Kolmogorov [5]. 
$d_{t}^{(n)}$ from some subinterval of $I$ to a line $h^{(n)}$ in $(b)$. Then for a subinterval $J$ of $I,\left\{d_{t}^{(n)}\right\}_{t \in J}$ omits a point $p$ of $h^{(n)}$. But $D$ is homomorphic to $h^{(n)}$ with $p$ removed and hence contains a connected set, the image of $\left\{d_{t}^{(n)}\right\}_{t \in J}$ under such a homomorphism. Since a locally compact division ring is readily seen to be either connected or totally disconnected we have

LEMMA 2.11. $D$ is connected.

It follows now from Pontrjagin's theorem that $D$ is the real, complex or quaternion division ring. ${ }^{6}$ We assume henceforward that the real or complex case has been singled out, e.g., by the assumption of simple ordering on the one hand or algebraic closure on the other, the quaternions having been set aside by postulating commutativity for $D$, i.e., that Pappus's theorem holds under finite elements. Turning now to the representation of $P$ itself, we shall need the final postulate

(C) For each $i=0,1, \cdots, P_{i}$ is complete. ${ }^{\text {? }}$

LEMMA 2.12. Let $L$ and $A$ be complete weakly modular lattices and let $L_{0}\left(\Lambda_{0}\right)$ be a subset of $L(\Lambda)$ such that every element of $L(\Lambda)$ is a join of elements of $L_{0}\left(\Lambda_{0}\right)$. Suppose further that $\phi$ is a mapping of $L_{0}$ onto $\Lambda_{0}$ such that

(1) $a \perp b$ if and only if $\varphi(a) \perp \varphi(b)$.

Then $\varphi$ can be extended to an isomorphism of $L$ onto $A$.

Define $\theta: L \rightarrow \Lambda$ by $\theta(a)=\bigvee \varphi(c): c \in[a]$ where $[a]=\left\{b \leqq a: b \in L_{0}\right\}$. Clearly $\theta$ preserves order and $\theta \mid L_{0}=\varphi$. The lemma is proved in the following steps:

(2) $\theta\left(a^{\prime}\right) \leqq \theta(\alpha)^{\prime}$.

(3) $a<b$ implies $\theta(a)<\theta(b)$.

(4) Let $A$ be a subset of $[a]$ such that $a=\sup A$. Then $\theta(a)=$ $\sup \varphi(b): b \in A$.

(5) $\theta(a \vee b)=\theta(a) \vee \theta(b)$.

(6) $\theta$ is one-to-one.

(7) $\theta^{-1}$ preserves order.

(8) $\theta$ is onto.

The proofs are as follows.

(2) If $b \in\left[a^{\prime}\right]$ and $c \in[a], \varphi(b) \leqq \varphi(c)^{\prime}$ by (1) so $\theta\left(a^{\prime}\right)=\bigvee \varphi(b): b \in\left[a^{\prime}\right] \leqq$ $\Lambda \varphi(c)^{\prime}: c \in[a]=(\bigvee \varphi(c))^{\prime}: c \in[a]=\theta(a)^{\prime}$.

(3) If $a<b$, there exists $c \neq 0 \varepsilon\left[b a^{\prime}\right]$. Then $\varphi(c) \perp \varphi\left(a_{1}\right)$ for all $a_{1} \in[a]$ by (1) so $\varphi(c) \perp \theta(a)$. Clearly $\varphi(c) \leqq \theta(b)$ and $\theta(a) \leqq \theta(b)$ so $\theta(a)<$ $\theta(b)$.

(4) Let $\alpha=\sup \varphi(b): b \in A$; clearly $\alpha \leqq \theta(a)$. If $c \in L_{0}$ with $\varphi(c) \in$ $\left[\theta(a) \alpha^{\prime}\right]$ then $c \perp b$ for every $b \in A$ by (1) so $c \perp a$. Hence $\varphi(c) \leqq$

${ }^{6}$ See $[8]$ for a unified derivation of the classification of locally compact division rings.

${ }^{7}$ Cf. the remark following the statement of postulate $\left(C^{\prime}\right)$. 
$\theta\left(a^{\prime}\right) \leqq \theta(\alpha)^{\prime}$ by $(2)$. Since $\varphi(c) \leqq \theta(\alpha), c=0$ and hence $\theta(a)=\alpha$ by weak modularity.

(5) Let $A=[a] \cup[b]$. Then $\sup A=a \vee b$ so $\theta(a \vee b)=\sup \varphi(c): c \in A$ by (4). Now if $c \in A, \varphi(c) \leqq \theta(a)$ or $\varphi(c) \leqq \theta(b)$ so $\mathcal{P}(c) \leqq \theta(a) \vee \theta(b)$; the opposite inequality is immediate.

( 6 ) and ( 7 ). If $a \$ b$ then $b<a \vee b$ so $\theta(b)<\theta(a \vee b)$ by $(3)=\theta(a) \vee \theta(b)$ by (5) and hence $\theta(a) \$ \theta(b)$.

(8) Let $\alpha \in \Lambda$ and let $A=\left\{\mathscr{\rho}^{-1}(\beta): \beta \in[\alpha]\right\}$. Then, by (4), $\theta(\sup A)=$ $\mathrm{V} \beta: \beta \in[\alpha]=\alpha$.

For each $a \in P_{f}$ we choose a distinct Euclidean space $H_{a}$ over $D$ of dimension $1+\operatorname{dim} a$ and an isomorphism $\varphi_{a}$ of $(a)$ onto $L_{a}$, the lattice of subspaces of $H_{a}$. Assuming $n=\operatorname{dim} a>0$, we wish to choose a scalar product for $H_{a}$ so that the orthocomplementation $\mathcal{P}_{a} b \rightarrow \mathcal{P}_{a} a b^{\prime}$ which is induced in $L_{a}$ by that in $(a)$ coincides with the one induced by the scalar product. First of all, there exists an involution $\sigma$ of $D$ and non-zero numbers (i.e., elements of $D) \gamma_{0}, \cdots, \gamma_{n}$ such that $\gamma_{i}^{\tau}=\gamma_{i}$, $\sum_{i=0}^{n} x_{i} \gamma_{i} x_{i}^{\sigma}=0$ implies all $x_{i}=0$, and if $b \in(a)_{0}$ and $\varphi_{a} b=\left[\left(x_{0}, \cdots, x_{n}\right)\right]$ then $\phi_{a} a b^{\prime}=\left\{\left(y_{0}, \cdots, y_{n}\right): \sum y_{i} \gamma_{i} x_{i}^{\tau}=0\right\} .^{8}$ In the real case, $\sigma=1$ is the only automorphism; we shall show that $\sigma$ is continuous, hence is either 1 or conjugation in the complex case, and the value 1 is excluded, for otherwise $\left(\gamma_{0}^{-1 / 2},\left(-\gamma_{1}^{-1 / 2}\right), 0, \cdots, 0\right)$ would be self-orthogonal. Then all the $\gamma_{i}$ must be positive real numbers and the desired scalar product is $(y, z)=\sum y_{i} \gamma_{i} \bar{z}_{i}$.

Let $b$ and $c$ be orthogonal points in $(a)$, and choose $x, y$ in $H_{a}$ such that $\varphi_{a} b=[x], \varphi_{a} c=[y]$. Let $\lambda_{m}$ be a sequence of numbers with $\lambda_{m} \rightarrow 0$ and let $b_{m}=\varphi_{a}^{-1}\left[x+\lambda_{m} y\right]$. Then $b_{m} \rightarrow b$ so $(b \vee c) b_{m}^{\prime} \rightarrow(b \vee c) b^{\prime}=c$ by Lemma 2.8 and we may assume that $(b \vee c) b_{m}^{\prime} \neq b$ holds for all $m$. Then a sequence $\mu_{m}$ of numbers with $\mu_{m} \rightarrow 0$ is determined by: $\varphi_{a}(b \vee c) b_{m}^{\prime}=$ $\left[\mu_{m} x+y\right]$. Since $b \perp c, \sum\left(\mu_{m} x_{i}+y_{i}\right) \gamma_{i}\left(x_{i}+\lambda_{m} y_{i}\right)^{\sigma}=0$ so $0=\mu_{m} \sum x_{i} \gamma_{i} x_{i}^{r}+$ $\lambda_{m}^{\sigma} \sum y_{i} \gamma_{i} y_{i} y_{i}^{\sigma}$ and it follows from the fact that $\mu_{m} \rightarrow 0$ and $\sum y_{i} \gamma_{i} y_{i}^{\sigma} \neq 0$ that $\lambda_{m}^{\sigma} \rightarrow 0$. Thus, $\sigma$ is continuous at 0 and hence, by its additivity, is continuous everywhere, and the proof is complete.

We assume now, in accordance with the foregoing, that each $H_{a}$ has been provided with a scalar product such that $\varphi_{a} b \perp \varphi_{a} c$ for $b, c$ in (a) if and only if $b \perp c$. If $a \leqq b \in P_{f}, \varphi_{b a}=\varphi_{b} \varphi_{a}^{-1}$ is clearly an orthogonality preserving isomorphism of $L_{a}$ in $L_{b}$. It is well known that there then exists an isometric transformation $\psi_{b a}$ of $H_{a}$ in $H_{b}$, unique up to multiplication by a number of absolute value one, such that if $v \in H_{a}$, $\phi_{b a}[v]=\left[\psi_{b a} v\right]$. We shall show that the $\psi^{\prime}$ 's may be chosen consistently, i.e., so that

(15) $a \leqq b \leqq c$ implies $\psi_{c a}=\psi_{c b} \psi_{b a}$.

We establish a one-to-one correspondence $\alpha \leftrightarrow a_{a}$ between the elements

${ }^{8}\left[\mathbf{2}\right.$, Appendix]. $\left[\left(x_{0}, \cdots, x_{n}\right)\right]$ denotes the 1-dimensional subspace of $H_{x}$ generated by the element $\left(x_{0}, \cdots, x_{n}\right)$. 
of $P_{f}$ and the ordinal numbers less than an ordinal $\zeta$ such that $\alpha<\beta$ implies $\operatorname{dim} a_{a} \leqq \operatorname{dim} a_{\beta}$. Thus, in particular, $a_{0}=0$; it is understood that all ordinals $\alpha, \beta, \cdots$ which occur lie under $\zeta$ and, where no confusion can result, we shall write " $\alpha$ " for " $a_{\alpha}$ ". In particular, we let $\alpha \beta$ represent (the index of) $a_{\alpha} a_{\beta}$. Let $\gamma<\zeta$ and suppose that $\psi$ has already been defined so that (15) holds for $c=a_{\alpha}$ with $\alpha<\gamma$. Now choose $\alpha$ such that $a_{\alpha}<a_{\gamma}$ and $\operatorname{dim} a_{\gamma}-\operatorname{dim} a_{\alpha}=1$; we call such an $\alpha$ "maximal". Fix $\psi_{\gamma, \alpha}$ arbitrarily; then if $a_{\eta}<a_{\alpha}, \psi_{\gamma, \eta}$ is defined as $\psi_{\gamma, \alpha} \psi_{\alpha, \eta}$. If $\beta$ is a second maximal element, and assuming $\operatorname{dim} a_{\gamma}>1$ (i.e., $\operatorname{dim} H_{\gamma}>2$ ), for otherwise there is nothing to prove, $\alpha \beta \neq 0$ and we define $\psi_{\gamma, \beta}$ by $\psi_{\gamma, \alpha \beta}=\psi_{\gamma, \beta} \psi_{\beta, \alpha \beta}$. Now let $\eta$ be any ordinal with $a_{\eta}<a_{\gamma}$ and let $\beta, \varepsilon$ both be maximal such that $a_{\eta} \leqq a_{\beta}$ and $a_{\eta} \leqq a_{\varepsilon}$. Assuming that $\operatorname{dim} a_{\gamma} \geqq 3$, we shall show

(16) $\psi_{\gamma, \beta} \psi_{\beta, \eta}=\psi_{\gamma, \varepsilon} \psi_{\varepsilon, \eta}\left(\beta, \varepsilon \operatorname{maximal}, a_{\eta} \leqq a_{\beta \varepsilon}, \operatorname{dim} a_{\gamma} \geqq 3\right)$.

But if (16) holds for $\eta=\beta \varepsilon$ then, by the inductive hypothesis, it will hold for arbitrary $\eta$, for then $\psi_{\gamma, \beta} \psi_{\beta, \eta}=\psi_{\gamma, \beta} \psi_{\beta, \beta \varepsilon} \psi_{\beta \varepsilon, \eta}=\psi_{\gamma, \varepsilon} \psi_{\varepsilon, \beta \varepsilon} \psi_{\beta \varepsilon, \eta}=$ $\psi_{\gamma, \varepsilon} \psi_{\varepsilon, \eta}$. To prove (16) in the case $\eta=\beta \varepsilon$ observe that $\psi_{\gamma, \alpha} \psi_{\alpha, \alpha \beta \varepsilon}=$ $\psi_{\gamma, \alpha} \psi_{\alpha, \alpha \beta} \psi_{\alpha \beta, \alpha \beta \varepsilon}=\psi_{\gamma, \alpha \beta} \psi_{\alpha \beta, \alpha \beta \varepsilon}=\psi_{\gamma, \beta} \psi_{\beta, \alpha \beta} \psi_{\alpha \beta, \alpha \beta \varepsilon}=\psi_{\gamma, \beta} \psi_{\beta, \alpha \beta \varepsilon}=\psi_{\gamma, \beta} \psi_{\beta, \beta \varepsilon} \psi_{\beta \varepsilon, \alpha \beta \varepsilon}$. Similarly-interchanging $\beta$ and $\varepsilon-\psi_{\gamma, \alpha} \psi_{\alpha, \alpha \beta \varepsilon}=\psi_{\gamma, \varepsilon} \psi_{r_{\varepsilon}, \beta \varepsilon} \psi_{\beta \varepsilon, \alpha \beta \varepsilon}$. In other words, $\psi_{\gamma, \varepsilon} \psi_{\varepsilon, \beta \varepsilon}=\psi_{\gamma, \beta} \psi_{\beta, \beta \varepsilon}$ on $\psi_{\beta \varepsilon, \alpha \beta \varepsilon} H_{\alpha \beta \varepsilon}$ and since $\alpha \beta \varepsilon \neq 0$ (by our assumption that $\operatorname{dim} a_{\gamma} \geqq 3$ ), this equality holds on all of $H_{\beta \varepsilon}$ and (16) is proved. Thus, if $\beta$ is maximal and $a_{\eta}<a_{\beta}, \psi_{\gamma, \eta}$ is unambiguously defined by: $\psi_{\gamma, \eta}=\psi_{\gamma, \beta} \psi_{\beta, \eta}$. If $a_{\eta}<a_{\delta}<a_{\gamma}$, choose $\beta$ maximal with $a_{\delta}<a_{\beta}$ and then $\psi_{\gamma, \eta}=\psi_{\gamma, \beta} \psi_{\beta, \eta}=\psi_{\gamma, \beta} \psi_{\beta, \delta} \psi_{\delta, \eta}=\psi_{\gamma, \delta} \psi_{\delta, \eta}$, completing the proof that $\psi$ as extended to all $\gamma, \eta$ with $a_{\eta}<a_{\gamma}$ satisfies (15) providing that $\operatorname{dim} a_{\gamma} \geqq 3$. We begin the induction and complete the proof by "constructing" all $\psi_{c, b}$ with $\operatorname{dim} c \leqq 2$ in the following way. Let $A_{i}$ denote the set of all $\alpha<\zeta$ for which $\operatorname{dim} a_{\alpha}=i, i=0,1, \cdots$ Let $\beta_{1} \varepsilon A_{1}$, let $B_{1}=\left\{\beta \in A_{1}: \beta<\beta_{1}\right\}$ and make the inductive assumption that $\psi_{\gamma, \beta}$ and $\psi_{\beta, \alpha}$ (and consequently $\psi_{\gamma, \alpha}$ ) have already been consistently defined whenever $\beta \in B_{1}, \gamma \in A_{2}, \alpha \in A_{0}$ and $a_{\alpha}<a_{\beta}<a_{\gamma}$. For all $\gamma \in A_{2}$ such that $a_{\beta_{1}}<a_{\gamma}$, define $\psi_{\gamma, \beta_{1}}$ arbitrarily and then, choosing $\alpha \in A_{0}$ with $a_{\alpha}<a_{\beta_{1}}$, define $\psi_{\beta_{1}, \alpha}$ by $\psi_{\gamma, \alpha}=\psi_{\gamma, \beta_{1}} \psi_{\beta_{1}, \alpha}$ if $\psi_{\gamma, \alpha}$ has already been defined for some $\gamma \in A_{2}$ with $a_{\alpha}<a_{\gamma}$-i.e., if $a_{\alpha}<a_{\beta}<a_{\gamma}$ for some $\beta \in B_{1}$; otherwise define $\psi_{\beta_{1}, \alpha}$ arbitrarily and set $\psi_{\gamma, \alpha}=\psi_{\gamma, \beta_{1}} \psi_{\beta_{1}, \alpha}$ for all $\gamma \in A_{2}$ with $a_{\beta_{1}}<a_{\gamma}$. This procedure evidently extends $\psi$ consistently to all $\gamma, \beta_{1} ; \beta_{1}, \alpha$ and $\gamma, \alpha$ such that $\gamma \in A_{2}, \alpha \in A_{0}$ and $a_{\alpha}<a_{\beta_{1}}<a_{\gamma}$. It then follows inductively-beginning with $B_{1}=\phi$ - that $\psi$ may be consistently defined for all $\psi_{c, a}$ such that $a \leqq c$ and $\operatorname{dim} c \leqq 2$.

Now let $H$ be a separable, infinite dimensional Hilbert space over $D$, let $L$ be its lattice of closed subspaces and let $\left\{v_{i}\right\}$ be a complete orthonormal set in $H$. Let $\left\{a_{i}\right\}$ be a maximal orthogonal subset of $P_{0}$ which exists by Zorn's lemma and is countable by $\mathrm{E} 4$ and $(A)$, and for each $i$ let $u_{i}$ be a fixed unit vector in $H_{a_{i}}$. Let $a \in P_{0}$, let $u \in H_{a}$ and define 


$$
\begin{aligned}
\lambda_{i}(u) & =\left(\psi_{a \vee a_{i}, a} u, \psi_{a \vee a_{i}, a_{i}} u_{i}\right), \\
\xi u & =\sum \lambda_{i}(u) v_{i}, \\
\theta(a) & =\left\{\xi u: u \in H_{a}\right\} .
\end{aligned}
$$

Thus, the domain of $\lambda_{i}$ and $\xi$ is $\bigcup_{a \in P_{0}} H_{a}$, that of $\theta$ is $P_{0}$ and their ranges are in $D, H$, and the set $L_{0}$ of one dimensional subspaced of $H$ respectively. ${ }^{9}$ We shall show that $\theta$ is one-to-one, onto and that $\theta$ and $\theta^{-1}$ preserve orthogonality. Hence by Lemma 2.12, $\theta$ can be extended to an isomorphism $\bar{\theta}$ of $P$ on $L$. Then $f \bar{\theta}^{-1}$ will be a state for $L$ and the characterization of $\mathscr{S}$ is given by the $e^{10}$

Theorem of Gleason. ([5]) Let $\mu$ be a state on the lattice $L$ of closed subspaces of the separable real or complex Hilbert space $H$ of dimension at least three. Then there exists an orthonormal basis $\left\{x_{i}\right\}$ for $H$ and nonnegative real numbers $\lambda_{i}$ with $\sum \lambda_{i}=1$ such that if $Q$ is the projection on $M \in L, \mu(M)=\sum \lambda_{i}\left(Q x_{i}, x_{i}\right)$.

Each $L_{a}$ for $a \in P_{\rho}$ becomes a metric space under the definition: distance $\left(M_{1}, M_{2}\right)=\sup \left\{\left|\omega\left(M_{1}\right)-\omega\left(M_{2}\right)\right|: \omega\right.$ a state for $\left.L_{a}\right\}$. An immediate consequence of Gleason's theorem is that $\varphi_{a}$ is an isometry of $(a)$ on $L_{a}$.

Lemma 2.13. Let $a \in P_{0}, u \in H_{a}$. Then $\|\xi u\|=\|u\|$.

Proof. For $n=1,2, \cdots$ let $b_{n}=a \vee a_{1} \vee \cdots \vee a_{n}$. Then if $1 \leqq$ $i \leqq n$,

$$
\begin{aligned}
\lambda_{i}(u) & =\left(\psi_{a \vee a_{i}, a} u, \psi_{a \vee a_{i}, a} u_{i}\right) \\
& =\left(\psi_{b_{n}, a \vee a_{i}} \psi_{a \vee a_{i}, a} u, \psi_{b_{n}, a \vee a_{i}} \psi_{a \vee a_{i}, a_{i}} u_{i}\right) \\
& =\left(\psi_{b_{n}, a} u, \psi_{b_{n}, a_{i}} u_{i}\right) \text { so } \sum_{i=1}^{n}\left|\lambda_{i}(u)\right|^{2} \leqq\left\|\psi_{b_{n}, a} u\right\|^{2} \\
& =\|u\|^{2} \text { since the } \psi_{b_{n}, a} u_{i} \text { are orthonormal in } H_{b_{n}} \text { and } \psi_{b_{n}, a} \text { is an isometry. }
\end{aligned}
$$

Since $\xi$ is linear, we assume without essential loss of generality that $\|u\|=1$ and suppose that, contrary to the assertion of the lemma, $\|\xi u\|=\left(\sum_{i=1}^{\infty}\left|\lambda_{i}(u)\right|^{2}\right)^{1 / 2}=\delta<1$. Then, in particular, $\left(\psi_{b_{n}, a_{i}} u_{i}\right)_{i=1}^{n}$ must fail to be a basis in all but a finite number of the $H_{b_{n}}$, so, for convenience, we assume $b_{n}>a_{1} \vee \cdots \vee a_{n}$ for all $n$ and let $c_{n}=b_{n} a_{i}^{\prime} \cdots a_{n}^{\prime}$; evidently, $c_{n} \in\left(b_{n}\right)_{0}$. Let $w_{n}=\sum_{i=1}^{n} \lambda_{i}(u) \psi_{b_{n}, a_{i}}$, let $\alpha_{n}=\left\|\psi_{b_{n}, a} u-w_{n}\right\|$ and let $y_{n}=\left(\psi_{b_{n}, a} u-w_{n}\right) \alpha_{n}^{-1}$. Clearly $\alpha_{n} \rightarrow \sqrt{1-\delta^{2}}$ and $y_{n} \in \varphi_{b_{n} c_{n}}$. Then if $n>m$,

$$
\left(y_{n}, \psi_{b_{n}, b_{m}} y_{m}\right)=\alpha_{n}^{-1} \alpha_{m}^{-1}\left(\psi_{b_{n}, a} u-w_{n}, \psi_{b_{n}, a} u-\psi_{b_{n}, b_{m}} w_{m}\right)
$$

\footnotetext{
${ }^{9}$ For the convergence of $\xi u$, see the proof of Lemma 2.13 .

${ }^{10}$ It follows then from S1 and $\mathrm{S} 2$ that $\mathscr{S}$ contains all states for $P$.
} 


$$
\begin{aligned}
& =\alpha_{n}^{-1} \alpha_{m}^{-1}\left(\left\|\psi_{b_{n}, a} u\right\|^{2}-\left(w_{n}, \psi_{b_{\hat{n}}, a} u\right)-\left(\psi_{b_{n}, a} u, \psi_{b_{n}, b_{m}} w_{m}\right)+\left(w_{n}, \psi_{b_{n}, b_{m}} w_{m}\right)\right) \\
& \left.=\alpha_{n}^{-1} \alpha_{m}^{-1}\left(1-\sum_{i=1}^{n}\left|\lambda_{i}\right|^{2}\right)-\sum_{i=1}^{m}\left|\lambda_{i}\right|^{2}+\sum_{i=1}^{m}\left|\lambda_{i}\right|^{2}\right) \\
& =\alpha_{n}^{-1} \alpha_{m}^{-1}\left(1-\sum_{i=1}^{n}\left|\lambda_{i}\right|^{2}\right) \rightarrow \frac{1-\delta^{2}}{1-\delta^{2}}=1 .
\end{aligned}
$$

Thus, given $\varepsilon>0$, we may choose $N$ so that $n>m>N$ implies $\varepsilon>$ $1-\left(y_{n}, \psi_{b_{n}, b_{m}} y_{m}\right)=\operatorname{distance}\left(\left[y_{n}\right],\left[\psi_{b_{n}, b_{m}} y_{m}\right]\right)=\rho\left(c_{n}, c_{m}\right)$. Then, in virture of (C), there exists a point $c$ in $P$ such that $c_{n} \rightarrow c$. We shall complete the proof by showing that $c \perp a_{i}$ for all $i$ contrary to the maximality of $\left\{a_{i}\right\}$. Indeed, if $f \in \mathscr{S}$ with $f\left(a_{i}\right)=1$ and $n>i$, then $c_{n} \perp a_{i}$, and if $n$ is chosen so large that $\rho\left(c_{n}, c\right)$ is less than a preassigned $\varepsilon>0$, $f(c)<f\left(c_{n}\right)+\varepsilon=\varepsilon$, i.e., $f(c)=0$ so $c \perp a_{i}$ by (6) of Theorem 1.1 and the proof of Lemma 2.13 is complete.

CoRollary 1. Let $a$ and $b$ be points. Then $\theta a \perp \theta b$ if and only if $a \perp b$.

Proof. For $u \in H_{a \vee b}$ let $\eta u=\sum\left(\psi_{a \vee b \vee a_{i}, a \vee b} u, \psi_{a \vee b \vee a_{i}, a_{i}} u_{i}\right) v_{i}$. Clearly $\eta$ is linear and if $c=\varphi_{a \vee b}^{-1}[u]$ and

$$
\begin{aligned}
w & =\psi_{c \vee a_{i}, c}^{-1} u, \eta u=\sum\left(\psi_{a \vee b \vee a_{i}, c \vee a_{i}} \psi_{c \vee a_{i}, c} w, \psi_{a \vee b \vee a_{i}, c \vee a_{i}} \psi_{c \vee a_{i}, a_{i}} u_{i}\right) v_{i} \\
& =\sum\left(\psi_{c \vee a_{i}, c} w, \psi_{c \vee a_{i}, a_{i}} u_{i}\right) v_{i}=\xi w ;
\end{aligned}
$$

clearly $\theta c=[\eta u]$. Hence $\|\eta u\|=\|\xi w\|=\|w\|=\|u\|$ so $\eta$ is an isometry and then letting $0 \neq u \in \varphi_{a \vee b} a, 0 \neq v \in \varphi_{a \vee b} b, a \perp b$ if and only if $u \perp v$ if and only if $\eta u \perp \eta v$ if and only if $\theta a \perp \theta b$.

Corollary 2. $\theta$ is one-to-one.

Proof. If $\theta a=\theta b$ and $c \in\left(b^{\prime}\right)_{0}, c \perp b$ so $\theta c \perp \theta b$ by Corollary 1, $\theta c \perp \theta a$ by our assumption and then $a \leqq c^{\prime}$ by Corollary 1 . Hence $a \leqq \Lambda c^{\prime}: c \in\left(b^{\prime}\right)_{0}=\left(\bigvee c: c \in\left(b^{\prime}\right)_{0}\right)^{\prime}=b$ by postulate (A). Similarly $b \leqq a$, so $a=b$.

CoRollay 3. Let $b_{1}<b_{2}<\cdots$ be a chain of finite elements and suppose $y_{n} \in H_{b_{n}}$ with $\left\|y_{n}\right\|=1$ such that given $\varepsilon>0$ there exists $N$ such that $n>m>N$ implies $\left\|y_{n}-\psi_{b_{n}, b_{m}} y_{m}\right\|<\varepsilon$. Let $c_{n}=\phi_{b_{n}}^{-1}\left[y_{n}\right]$. Then the sequence of points $\left\{c_{n}\right\}$ converges to a point $c$ in $P$.

Lemma 2.14. $\theta$ is onto.

Proof. Let $M \in L_{0}, v=\Sigma \mu_{i} v_{i}$ a unit vector in $M$. Let $b_{n}=a_{1} \vee \cdots \vee a_{n}$, $w_{n}=\sum_{i=1}^{n} \mu_{i} \psi_{b_{n}, a_{i}} u_{i}, y_{n}=w_{n} /\left\|w_{n}\right\|$ when $w_{n} \neq 0$ and $c_{n}=\phi_{b_{n}}^{-1}\left[y_{n}\right]$. It 
follows at once from Corollary 3 above that there exists $c \in P_{0}$ with $c_{n} \rightarrow c$. Let $d_{n}=c \vee b_{n}$ and let $y$ be a unit vector in $H_{c}$. Now $\left(\psi_{a_{n}, b_{n}} y_{n}, \psi_{a_{n}, c} y\right)$ tends to a limit $\eta$ with $|\eta|=1$ and $\left\|\psi_{a_{n}, b_{n}} y_{n}-\eta \psi_{a_{n}, c} y\right\| \rightarrow 0$. Hence, by Lemma 2.13, $\xi y_{n} \rightarrow \xi \eta y$. Since $\xi y_{n} \rightarrow v$ is obvious, $\xi \eta y=v$, $\theta c=M$ and the proof is complete.

$\theta$ is one-to-one from $P_{0}$ onto $L_{0}$ by Corollary 2 of Lemma 2.13 and the preceding lemma. Furthermore, $\theta a \perp \theta b$ if and only if $a \perp b$ by Corollary 1 of Lemma 2.13 and so we may apply Lemma 2.12 to obtain

Theorem 2.2. Suppose the system $\mathscr{S}, P$ satis fies the following eight postulates:

(A) $P$ is atomic; $1 \notin P_{f}$.

(M) If $a$ is finite and $b, c$ and $d$ are elements of $(a)$ such that $d \leqq c$ and $b c=0$, then $(d \vee b) c=d$.

(H) If $a$ and $b$ are finite elements of the same dimension, then $(a)$ and (b) are isomorphic.

(ND) $P$ is not deterministic.

(C') If $a$ is finite and $0 \leqq i \leqq \operatorname{dim} a,(a)_{i}$ is compact.

(Co) There exists a continuous, non-constant function from an interval of the real line to the lattice under a finite event.

(P) If $a$ is finite, Pappus's theorem holds in (a).

(C) For each $i=0,1, \cdots, P_{i}$ is complete.

Then $P$ is isomorphic to the lattice $L$ of closed subspaces of a separable, infinite dimensional Hilbert space over either the real or the complex field in such a way that the orthocomplementations in $P$ and $L$ correspond.

\section{REFERENCES}

1. G. Birkhoff, Lattice theory, Amer. Math. Soc. Colloq. Publ., 25, revised, 1948.

2. G. Birkhoff and J. von Neumann, The logic of quantum mechanics, Ann. of Math., 37 (1936), 823-843.

3. A. M. Gleason, Measures on the closed subspaces of a Hilbert space, J. of Rat. Mech. and Analysis, 6 (1957), 885-894.

4. S. Kakutani, Concrete representation of abstract $(L)$-spaces and the mean ergodic theorem, Ann. of Math., 42 (1941), 523-537.

5. A. Kolmogorov, Zur Begründung der projektiven Geometrie, Ann. of Math., 33 (1932), 175-176.

6. G. W. Mackey, Lecture notes for Math. 265, The mathematical foundations of quantum mechanics, Harvard, Spring '55-'56 (unpbl.).

7. G. W. Mackey, Quantum mechanics and Hilbert space, Amer. Math. Monthly 64, no. 8, part II (1957), 45-57.

8. E. Weiss and N. Zierler, Locally compact division rings, Pacific J. Math., 8 (1958), 369371.

Jet Propulsion Laboratory, California Institute of TeChNology 
